\title{
Charge Carrier Transport in Quantum Cascade Lasers in Strong Magnetic Field
}

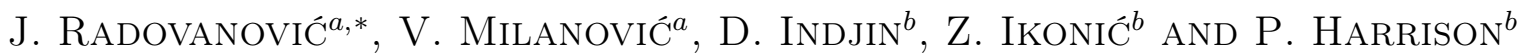
${ }^{a}$ School of Electrical Engineering, University of Belgrade, Bulevar kralja Aleksandra 73, 11120 Belgrade, Serbia

${ }^{b}$ Institute of Microwaves and Photonics, School of Electronic and Electrical Engineering, University of Leeds

Woodhouse Lane, Leeds, LS2 9JT, UK

We have developed a comprehensive rate equations based model for calculating the optical gain in the active region of a quantum cascade laser in magnetic field perpendicular to the structure layers, which takes into account all the relevant scattering channels. The model is applied to gain-optimized quantum cascade laser active region, obtained by a systematic optimization procedure based on the use of genetic algorithm, which we have previously set up for designing novel structures and improving performance of existing ones. It has proven to be very efficient in generating optimal structures which emit radiation at specified wavelengths corresponding to absorption fingerprints of particular harmful pollutants found in the atmosphere. We also illustrate another interesting prospective application of quantum cascade laser-type structures: the design of metamaterials with tunable complex permittivity, based on amplification via intersubband transitions. In this case, the role of the magnetic field is to assist the attainment of sufficient optical gain (population inversion), necessary to effectively manipulate the permittivity and fulfill the conditions for negative refraction (left-handedness).
\end{abstract}

PACS: 72.10.-d, 73.21.Fg

\section{Introduction}

The mechanism of photon generation in quantum cascade laser (QCL) is based on transitions between the quasi-bound states which are associated with an ultrashort excited state lifetime corresponding to electronlongitudinal optical (LO) phonon scattering [1]. Application of a strong magnetic field has proven to be a sensitive instrument for studying and controlling these intersubband processes as it provides a path for modulating the laser output properties [2-6]. In effect, the field introduces additional quantization of the in-plane electron motion and creates a series of the Landau levels (LLs) instead of two-dimensional subbands, which resembles a quantum box structure. LLs are magnetically tunable and, depending on their configuration, phonon emission is either inhibited or resonantly enhanced. This leads to a strong modulation of the population inversion and consequently the optical gain, as a function of magnetic field.

We have previously developed an automated design procedure for structural parameters optimization of the active region of mid-infrared quantum cascade laser, with the goal of maximizing the output properties, in particular the optical gain, at predefined wavelengths compliant with selected application [4-8]. In mid-infrared devices, the desired emission wavelength imposes the required separations between the active laser energy states, while the spacing between the lower laser level and the ground state is set by the LO phonon energy (which facilitates the population inversion by allowing the fast emptying of the lower laser state by means of nonradiative transitions). The relationships between parameters of interest are very complex, making the optimization process

\footnotetext{
* corresponding author; e-mail: radovanovic@etf.bg.ac.rs
}

difficult and demanding, therefore a carefully selected optimization technique must be employed to perform this task, such as the genetic algorithm (GA) for global optimization $[7,9]$. Upon obtaining a gain-maximized structure, we introduce a strong external magnetic field parallel to the growth axis, to investigate the electron relaxation processes under three-dimensional confinement.

In this paper, we illustrate the application of our method for optimization of the active region of GaAs $/ \mathrm{Al}_{x} \mathrm{Ga}_{1-x} \mathrm{As}$ based mid-infrared QCLs. The GaAs/AlGaAs system was chosen because it is well understood from the material-growth point of view, and is virtually strain-free. In addition, the material parameters relevant for the effects considered in this work (especially the band nonparabolicity coefficients) are known more accurately, however, the presented numerical procedure is practically independent of the material system. Strong effects of band nonparabolicity [10] result in subtle changes of the lasing wavelength at magnetic fields which maximize the gain, thus providing a path for fine-tuning of the wavelength of output radiation. Another prospective application of QCL immersed in intense magnetic field is for the design of novel (active) metamaterials where optical losses can be compensated by adding sufficient gain $[11,12]$. This would enable all-semiconductor metamaterial realization, which relies on using the well established technology of epitaxial growth and incorporation into existing optical semiconductor devices.

\section{Theoretical consideration}

In our analysis we have considered two types of structures. The first one has a classically designed active region with three coupled quantum wells (QWs), biased by an external electric field. This system has three energy subbands $(n=1,2,3)$, with the laser transition occurring between subbands $n=3$ and $n=2$, Fig. 1a. 
The second structure, known as the "double-LO phonon" structure, has an extra level, which forms a part of a "ladder" of three states being mutually separated by exactly one LO phonon energy. In view of that, the active subbands corresponding to the laser transition are now marked as $n=4$ and $n=3$, as presented in Fig. 1b. This design is predicted to have a decreased lower laser level carrier lifetime and consequently a higher degree of population inversion [8]. In both cases the active region is surrounded by suitable emitter/collector regions in form of superlattices, designed as the Bragg reflectors, which inject electrons into the upper laser state on one side, and allow for rapid extraction of carriers from the lowest subband on the other side.

In the absence of an external magnetic field, the electronic subbands from Fig. 1 have free particle-like energy dispersion in the direction parallel to the QW planes: $E_{n}\left(k_{\|}\right)=E_{n 0}+\hbar^{2} k_{\| \mid}^{2} / 2 m_{\| n}\left(E_{n 0}\right)$, where $m_{\| n}\left(E_{n 0}\right)$ is the energy-dependent in-plane effective mass, and $k_{\|}$is the in-plane wave vector $\left(E_{n 0}=E_{n}\left(k_{0}=0\right)\right)$. The nonradiative lifetime for the state $\left|3, k_{||}\right\rangle$(or $\left|4, k_{||}\right\rangle$) is limited by electron-LO-phonon scattering into the two (three) lower subbands of the active region. However, when the structure is subjected to a strong magnetic field $B$ in the direction of the growth axis (Fig. 1a), continuous subbands $E_{n}\left(k_{\|}\right)$transform into series of individual (strictly discrete) states, the energies of which (with band nonparabolicity included) are given by [13]: $E_{n, l}=E_{n}\left(k_{\|}=0\right)+(l+1 / 2) \hbar e B / m_{\| n}\left(E_{n 0}\right)-1 / 8\left[\left(8 l^{2}+\right.\right.$ $\left.8 l+5) \alpha_{1}^{\prime}+\left(l^{2}+l+1\right) \beta_{1}^{\prime}\right]\left(\hbar e B / m_{1}^{2}\right)^{2}$, where $l=0,1,2, \ldots$ is the Landau index and $m_{1}$ is the parabolic effective mass of the well material. The in-plane electron effective mass is here calculated as $m_{\|}\left(E_{n 0}\right)=m^{*}\left[1+\left(2 \alpha_{1}^{\prime}+\beta_{1}^{\prime}\right) E_{n 0}\right]$ (this provides the best agreement with the experimental results, as explained in $[4,5])$, where the nonparabolicity parameters $\alpha_{1}^{\prime}$ and $\beta_{1}^{\prime}$ are evaluated according to Ref. [13]. It is evident that the realistic effects of band nonparabolicity influence the energy separation between the levels relevant for the radiative transition, i.e. the lasing wavelength becomes dependent on the magnetic field. This allows for the shift of the emission wavelength by variations of the magnetic field. The values of $B$ which give rise to resonant LO phonon emission (and in consequence, the dramatic reduction of the degree of population inversion) are found by solving the equation: $E_{3[4], 0}-E_{n, l}=\hbar \omega_{\mathrm{LO}}($ with $n=1,2[, 3])$, where $\hbar \omega_{\mathrm{LO}}$ is the LO phonon energy.

Optical transitions in this system are allowed only between states with the same value of the Landau index, i.e. $(i, l) \rightarrow(f, l)$. Summing up over all LLs, we get the total gain on all transitions between LLs belonging to the upper laser subband $n=4[3]$ and the lower laser subband $n=3[2]$ of the QCL active region, which reads $[4,5,7]$ :

$$
\begin{aligned}
& g_{[4] 3,[3] 2}=\frac{2 e^{2} \pi^{2}}{n \varepsilon_{0}} \frac{\left|d_{4[3], 3[2]}\right|^{2}}{\lambda} \\
& \quad \times \sum_{l} \delta\left(E_{4[3], l}-E_{3[2], l}-\hbar \omega\right)\left(N_{S 4[3]}-N_{S 3[2]}\right),
\end{aligned}
$$

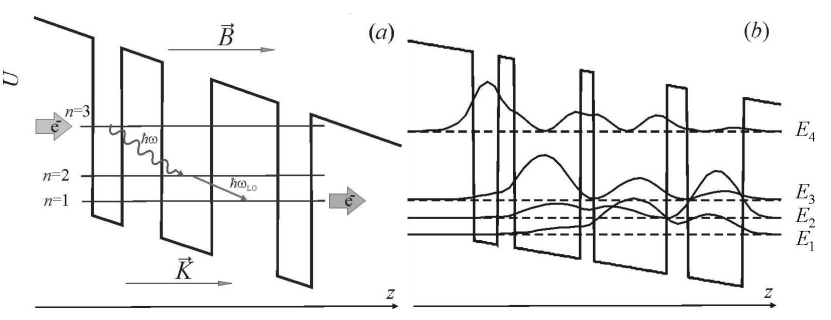

Fig. 1. (a) A schematic diagram of the triple-quantum-well QCL active region placed in perpendicular magnetic field, (b) the double-LO phonon structure described in the text, at zero magnetic field, with bound states wave functions squared.

where $n$ is the refractive index, $\varepsilon_{0}$ is the vacuum dielectric permittivity, $d_{\text {if }}$ is the transition matrix element calculated with $z$-dependent parts of the wave functions $\eta_{\mathrm{i}}$ and $\eta_{\mathrm{f}}$. Furthermore, $\lambda$ is the radiation wavelength, and $N_{S n}=\sum_{l} N_{n, l}$, where $N_{n, l}=e B /(\pi \hbar) F_{\mathrm{FD}}\left(E_{n, l}\right)$ is the electron areal density in the state $(n, l)$. To find the population inversion $N_{S 4}-N_{S 3}$ (or $N_{S 3}-N_{S 2}$ depending on the design of the active region), one has to find the electron distribution over all the states in the active region. This is obtained by solving the system of rate equations, which describe the change in level population as the difference between the rate at which the carriers arrive and the rate at which they leave. The exact form of the rate equations used in this work is presented in detail in references $[4,5,7]$.

In order to analyze the possibilities of constructing a novel metamaterial with the arrangement of structural layers as in quantum cascade laser, we must determine the dielectric permittivity tensor which may be written as [12]:

$$
\varepsilon=\left(\begin{array}{ccc}
\varepsilon_{\perp} & 0 & 0 \\
0 & \varepsilon_{\|} & 0 \\
0 & 0 & \varepsilon_{\|}
\end{array}\right), \quad \varepsilon_{\|}=\varepsilon_{\mathrm{b}} .
$$

This form is valid for quantum well based semiconductor nanostructures which exhibit strong anisotropy in optical properties. Here, $\varepsilon_{\|}$is the permittivity component along the QW planes, and is equal to the average permittivity of the background material $\left(\varepsilon_{\mathrm{b}}\right)$, while $\varepsilon_{\perp}$ describes the interaction along the growth $(z)$ axis and may be represented by the Lorentz model $[14,15]$ :

$$
\begin{aligned}
\varepsilon_{\perp} & =\varepsilon_{\mathrm{b}}+\frac{e^{2}}{\varepsilon_{0} L} \sum_{m<n}\left(N_{S_{m m}}-N_{S_{n n}}\right) \\
& \times \frac{\left|d_{m, n}\right|^{2}}{\left(\hbar \omega_{n m}-\hbar \omega\right)-\mathrm{i} \gamma_{n m}},
\end{aligned}
$$

where $L$ stands for the length of the unit cell in $z$ direction, $\omega_{n m}$ is the resonant transition frequency between states $n$ and $m$ and $\gamma_{n m}$ denotes the transition linewidth. $N_{S_{i i}}$ represents the electron sheet density in $i$-th state ( $N_{n, l}$ in the discretized spectrum). The magnetic permeability of a semiconductor-based nonmagnetic metamaterial is $\mu=1$, therefore, for this structure to behave 
as a left-handed metamaterial for transverse magnetic (TM) optical modes, the following conditions must be achieved [16]:

$$
\varepsilon_{\|}>0, \quad \operatorname{Re}\left(\varepsilon_{\perp}\right)<0 .
$$

As the first condition is automatically fulfilled, i.e. $\varepsilon_{\|}=\varepsilon_{\mathrm{b}}>0$, one clearly has to achieve high enough population inversion $N_{S_{n n}}>N_{S_{m m}}$ to cancel out the background term (which is both real and positive) and reverse the sign of $\operatorname{Re}\left(\varepsilon_{\perp}\right)$ in Eq. (3). The additional quantization introduced by external magnetic field applied to the QCL-type metamaterial, assists the realization of this objective for particular values of the field.

\section{Numerical results}

We first apply the advanced tools for global optimization, such as the simulated annealing or GA, to obtain structural parameters of gain-maximized quantum cascade laser emitting at specified wavelength. The GA is inspired by the theory of evolution, where problems are solved by selecting "the most capable" solution which is then allowed to survive. It begins with a set of solutions called population, which is used as a basis for generating another set of "offsprings" with best possible characteristics (defined by the value of the objective, i.e. the fitness function). This process is repeated until some predefined criterion is met (this could be, e.g. the total number of populations generated or the improvement in the fittest solution). One of the most important phases in the implementation of GA is the selection of a formal fitness function, which should be defined so to encompass all the goals of optimization. Here, the objective is to optimize the optical gain at selected wavelength, and the construction of the fitness function in this case is described in Ref. [7].

To illustrate the procedure, we have optimized the QCL active region at $\lambda \approx 7.3 \mu \mathrm{m}$ which corresponds to a characteristic line in the spectrum of sulphur-dioxide [17]. The structural parameters obtained by GA read: $11 \AA$, $32 \AA, 39 \AA, 23 \AA, 38 \AA$ (for the well and the barrier widths, respectively, going from left to right) and $U_{\mathrm{b}}=0.3175 \mathrm{eV}$ (the barrier height), which corresponds to aluminum mole fraction of $38 \%$, so the structure may be realized by $\mathrm{GaAs} / \mathrm{Al}_{0.38} \mathrm{Ga}_{0.62} \mathrm{As}$. The material parameters used in calculations are: $m^{*}=0.0665 m_{0}\left(m_{0}\right.$ is the free electron mass), $n=3.3$, and the conduction band discontinuity between GaAs and AlAs is $\Delta E_{\mathrm{c}}=$ $0.8355 \mathrm{eV}$. The applied electric field in the $z$-direction is $K=48 \mathrm{kV} / \mathrm{cm}$, and the minima of energy subbands are at $E_{1}\left(k_{\|}=0\right)=0.0828 \mathrm{eV}, E_{2}\left(k_{\|}=0\right)=0.1188 \mathrm{eV}$ and $E_{3}\left(k_{\|}=0\right)=0.2896 \mathrm{eV}$, as presented in Fig. 2a, together with the corresponding wave functions squared. Oscillations of the gain with $B$ are very pronounced, and prominent peaks are found at values of the magnetic field for which the resonant emission of LO phonons is inhibited so the lifetime of carriers in the upper laser state is increased. It is evident that the realistic effects of band nonparabolicity influence the energy separation between the levels relevant for the radiative transition, and this allows for the shift of the emission wavelength by variations of the magnetic field. The maximum of optical gain per unit of injection current is obtained at $B=34.5 \mathrm{~T}$, where the transition wavelength is shifted to $\lambda=7.52 \mu \mathrm{m}$, matching an absorption line in the spectrum of $\mathrm{HNO}_{3}$, while the peak second in magnitude, at $B=46 \mathrm{~T}$, may be used to monitor the presence of methane $\left(\mathrm{CH}_{4}\right)$ at $k=1320 \mathrm{~cm}^{-1}$ [17].
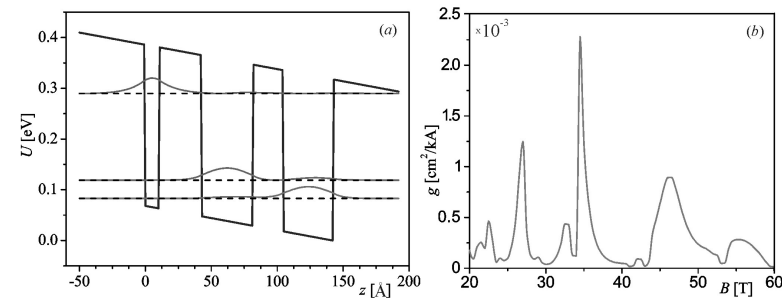

Fig. 2. (a) The active region of QCL, optimized for emission at $\lambda \approx 7.3 \mu \mathrm{m}$ which corresponds to a specific line in the spectrum of $\mathrm{SO}_{2}$. (b) The optical gain (per unit of injection current) as a function of the applied magnetic field, at $T=300 \mathrm{~K}$.

Next, we consider the influence of the magnetic field on electron relaxation processes in the optimized double-LO phonon $\mathrm{GaAs} / \mathrm{Al}_{0.45} \mathrm{Ga}_{0.55}$ As structure fully described in [8], obtained by simulated annealing algorithm. This structural profile represents the result of optimization of design parameters with the goal of enhancing the device performance. The layer widths are 46, 17, 11, 45, 8, 50, 14, 38 and $25 \AA$, going from emitter towards the collector barrier. The relaxation rates from the lowest LL of the upper laser level, taking into account LO and acoustic (AC) phonon induced transitions between the Landau levels, as well as electron-electron interactions [18] is presented in Fig. 3.

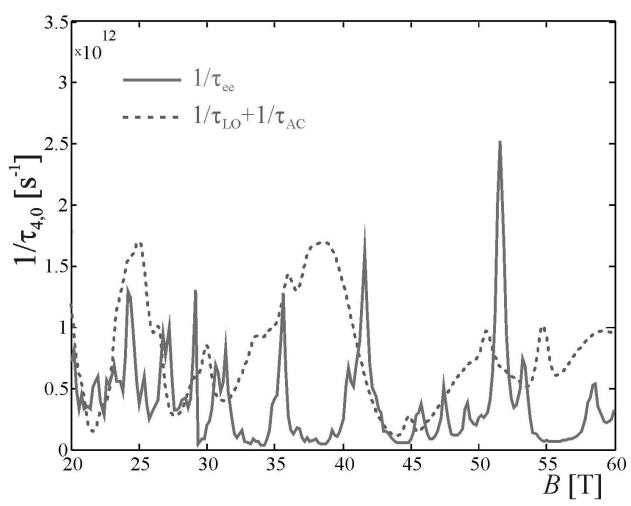

Fig. 3. Scattering rate diagram of electrons from the upper laser level, for the four-level QCL structure described in the text, due to electron-electron (solid line), and electron-phonon (dashed line) scattering.

In addition, it is interesting to analyze the possibilities of advanced dispersion engineering and design of ac- 
tive metamaterials based on high optical gain achieved with quantum cascade laser in a strong magnetic field. Numerical results obtained for QCL with active region from Fig. 2a illustrate significant potential for tuning of the sign and magnitude of the real and the imaginary part of the permittivity given by Eq. (3) with magnetic field. The dependence of $\varepsilon_{\perp}$ on radiation wavelength is calculated for magnetic fields which enhance the population inversion, keeping a fixed value of the injection current for all magnetic fields, Fig. 4. Due to the high background permittivity $\left(\varepsilon_{\mathrm{b}} \approx 13\right)$ it is necessary to reach very high values of the optical gain in order to obtain negative real part of the permittivity, and this can be accomplished only for large carrier sheet densities $\left(N_{S}=5 \times 10^{13} \mathrm{~cm}^{-2}\right)$. However, by applying this concept to other semiconductor material families (e.g. AlGaN/ $\mathrm{GaN}$ ), and combining it with a suitable optimization of structural parameters, it should be possible to reduce the level of gain required for switching between left-handed and (the conventional) right-handed regime.

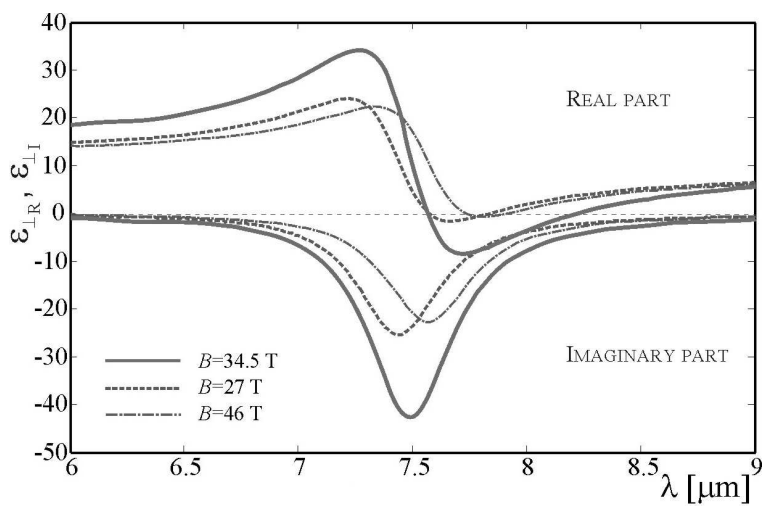

Fig. 4. The normal component of the total permittivity (the real and the imaginary part) of GaAs/AlGaAs QCL described in the text, as a function of the radiation wavelength, at magnetic fields which increase the population inversion. The carrier sheet density is $N_{S}=5 \times 10^{13} \mathrm{~cm}^{-2}$ and $T=300 \mathrm{~K}$.

\section{Conclusion}

This paper provides a brief overview of the results obtained by our modeling technique, developed for description of carrier transport in quantum cascade lasers placed in a strong external magnetic field. Within this method, electron distribution over the electronic states of the system is found by solving the full set of rate equations which describe the transitions between levels. As the Landau levels are magnetically tunable, depending on their configuration, phonon emission is either inhibited or resonantly enhanced. This translates into strong modulation of the population inversion and consequently the optical gain, as a function of magnetic field. The enhancement and suppression of the optical gain by magnetic field may be utilized to manipulate the effective medium parameters, namely the complex dielectric permittivity, and to construct novel semiconductor metamaterials with negative refraction. Numerical results are presented for different active regions types, obtained by global optimization procedures previously set up for the design of novel QCL structures emitting at selected wavelengths in the mid-infrared part of the spectrum.

\section{Acknowledgments}

This work was supported by the Ministry of Science (Republic of Serbia), ev. no. 141006. and by NATO Collaborative Linkage Grant (reference CBP.EAP.CLG 983316).

\section{References}

[1] J. Faist, F. Cappaso, D.L. Sivco, A.L. Hutchinson, A.Y. Cho, Science 264, 553 (1994).

[2] A. Vasanelli, A. Leuliet, C. Sirtori, A. Wade, G. Fedorov, D. Smirnov, G. Bastard, B. Vinter, M. Giovannini, J. Faist, Appl. Phys. Lett. 89, 172120 (2006).

[3] A. Wade, G. Fedorov, D. Smirnov, S. Kumar, B.S. Williams, Q. Hu, J.L. Reno, Nature Photon. 3, 41 (2009).

[4] J. Radovanović, A. Mirčetić, V. Milanović, Z. Ikonić, D. Indjin, P. Harrison, R.W. Kelsall, Semicond. Sci. Technol. 21, 215 (2006).

[5] J. Radovanović, V. Milanović, Z. Ikonić, D. Indjin, P. Harrison, J. Appl. Phys. 97, 103109 (2005).

[6] I. Savić, N. Vukmirović, Z. Ikonić, D. Indjin, R.W. Kelsall, P. Harrison, V. Milanović, Phys. Rev. B 76, 165310 (2007).

[7] A. Daničić, J. Radovanović, V. Milanović, D. Indjin, Z. Ikonić, J. Phys. D, Appl. Phys. 43, 045101 (2010).

[8] A. Mirčetić, D. Indjin, Z. Ikonić, P. Harrison, V. Milanović, R.W. Kelsall, J. Appl. Phys. 97, 084506 (2005).

[9] J. Radovanović, V. Milanović, Z. Ikonić, D. Indjin, J. Phys. D, Appl. Phys. 40, 5066 (2007).

[10] V. Milanović, J. Radovanović, S. Ramović, Phys. Lett. A 373, 3071 (2009).

[11] P. Ginzburg, M. Orenstein, J. Appl. Phys. 104, 063513 (2008).

[12] P. Ginzburg, M. Orenstein, J. Appl. Phys. 103, 083105 (2008).

[13] U. Ekenberg, Phys. Rev. B 40, 7714 (1989).

[14] P. Basu, Theory of Optical Processes in Semiconductors: Bulk and Microstructures, Clarendon Press, Oxford 1997.

[15] R.W. Boyd, Nonlinear Optics, 3rd ed, Academic Press, San Diego 2008.

[16] V.A. Podolskiy, E.E. Narimanov, Phys. Rev. B $\mathbf{7 1}$, 201101R (2005).

[17] www.hitran.com .

[18] B. Novaković, J. Radovanović, A. Mirčetić, V. Milanović, Z. Ikonić, D. Indjin, Opt. Commun. 279, 330 (2007). 